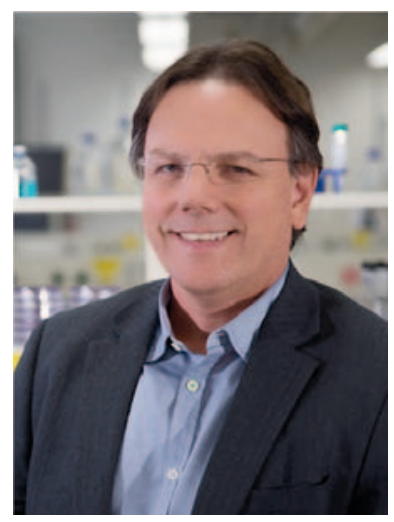

Prof. Dr. Dr. Gerhard Rogler, Zürich, Schweiz

\section{Komplementärmedizinische Ansätze in der Gastroenterologie}

Manchmal sind es Ereignisse oder Publikationen ausserhalb einer medizinischen Fachdisziplin, die deutlichen Einfluss nehmen und das Bild des Faches in der Bevölkerung und bei Patienten verändern. Der Darm und seine Funktionen sowie die Therapie seiner Erkrankungen waren über viele Jahrzehnte kein Thema, das im Fokus stand oder gar in der Öffentlichkeit diskutiert wurde. Das hat sich geändert seit der Publikation des Buches «Darm mit Charme» von Giulia Enders. Die wichtigen Funktionen des Darmes, aber auch seine Erkrankungen haben damit eine Öffentlichkeit erfahren, die zu vielen Nachfragen bei Ärztinnen und Ärzten geführt haben. Das passt in eine Zeit, in der sich viele Menschen über Ernährung Gedanken machen, aber auch tatsächliche und gefühlte Nahrungsmittelunverträglichkeiten dramatisch an Häufigkeit zunehmen. Dies hat nicht zuletzt zu einem Wildwuchs an Therapieempfehlungen bei Darmerkrankungen geführt, der Verwirrung gestiftet hat.

In den letzten Jahren ist auch das Darmmikrobiom bzw. die Darmmikrobiota in den Blickpunkt geraten. Während das Mikrobiom eigentlich nur die Gesamtheit der genetischen Information der Darmbakterien meint, bezeichnet Darmmikrobiota das gesamte Ökosystem (das früher fälschlicherweise «Darmflora» genannt wurde; Bakterien, Pilze und Viren sind jedoch keine Pflanzen). Bakterien erschienen uns über Jahrzehnte im Wesentlichen als Krank- heitserreger. Heute verstehen wir, dass das für 99\% der Bakterien im Darm nicht zutrifft. Ihre Funktionen sind essenziell für unsere Gesundheit. Die meisten diagnostischen Verfahren, die postulieren, die Zusammensetzung der Darmmikrobiota zu untersuchen, und auch mit Grenzwerten arbeiten, sind jedoch sehr störanfällig und sinnlos. Je nach Transportverfahren, Transportdauer und anderen Faktoren kann die Zusammensetzung der Darmmikrobiota in solchen Analysen extrem variieren. Auch «Pilze im Stuhl» sind völlig normal und kein Anlass zur Besorgnis.

Auf der Basis solcher fragwürdiger Tests für die Zusammensetzung der Darmmikrobiota wird eine Vielzahl von probiotischen Bakterienpräparationen zur Verbesserung der «Darmgesundheit» oder der «Immunfunktionen» angeboten. Die allermeisten dieser Marketing-Aussagen sind unbelegt und sollten sehr kritisch hinterfragt werden. Ein «Aufbau» einer «gesunden» Darmmikrobiota ist so nicht möglich.

Im Gegensatz dazu ist die Wirkung der fäkalen Mikrobiota-Transplantation (FMT) bei der Clostridien-Colitis gesichert und inzwischen im Grunde genommen medizinischer Standard. Die Erfolgsrate bei der rezidivierenden Clostridien-Infektion liegt in sehr gut publizierten Studien bei über 90\%, was wir aus eigener Erfahrung bestätigen können. Auch bei der Colitis ulcerosa scheint Potenzial für diese Therapie vorhanden zu sein. Erste Studien belegen einen Effekt der FMT. Ob sie aber bei den vielen anderen Erkrankungen helfen kann, für die sie momentan diskutiert wird (wie z.B. Diabetes oder Depressionen), ist sehr fraglich. Der Beitrag von Biedermann et al. [1] im Rahmen des Themenschwerpunktes «Gastroenterologie» in dieser Ausgabe der SchweIZERISCHEN ZeITSChrift FüR GANZHEITSMEDIZIN zeigt daher die gesicherten und ungesicherten Möglichkeiten der FMT auf und beschäftigt sich mit den Risiken.

Auf der Welle der Aufmerksamkeit für die Funktionen der Darmmikrobiota fand auch ein weiterer Therapieansatz wieder neues Interesse: die Darm-Detoxifikation. Hier muss man leider konstatieren, dass diese Massnahme häufig mehr Schaden als Nutzen bringt, da die Darmmikrobiota durchaus empfindlich gestört werden kann. Ohne klar nachgewiesenen Nutzen, jedoch mit signifikanten Risiken assoziiert wird dieser Ansatz im Artikel von Frei und Rogler [2] kritisch besprochen.

Einen klar nachgewiesenen Nutzen bei Entzündungen der Darmmukosa haben im Gegensatz dazu Anthocyane aus Beeren, Früchten und auch Gemüse sowie Curcuma. Curcuma wirkt über eine Inhibierung proentzündlicher

\section{KARGER}

(c) 2017 S. Karger GmbH, Freiburg

Fax +497614520714
Prof. Dr. Dr. Gerhard Rogler

Klinik für Gastroenterologie und Hepatologie

UniversitätsSpital Zürich

Rämistrasse 100, 8091 Zürich, Schweiz

gerhard.rogler@usz.ch 
zellulärer Faktoren wie NF- $\kappa$ B. Eine Wirkung von $3 \mathrm{~g}$ Curcumin täglich konnte kürzlich in einer placebokontrollierten, doppelblinden klinischen Studie bei Patientinnen und Patienten mit Colitis ulcerosa nachgewiesen werden. Auch für Anthocyane, die blauschwarzen oder rötlichen Farbstoffe, die wir aus Heidelbeeren, Aronia-Beeren, Brombeeren, Kirschen oder roten Trauben kennen, konnten antioxidative und antientzündliche Wirkungen nachgewiesen werden. Anthocyan-Präparationen werden bereits bei Venenentzündungen und der Zystitis (Cranberry-Extrakt) eingesetzt. Diesen beiden Substanzen widmet sich der Beitrag von Scharl et al. [3].

In den einzelnen Artikeln dieser Ausgabe möchten wir demonstrieren, dass es bei aller kritischen Distanz wirksame komplementärmedizinische Therapien im Bereich der Darmerkrankungen bereits gibt. Aufgrund der Studiendaten haben sie gute Chancen, sich als Standardtherapien zu etablieren. Andere Verfahren (Darm-Detoxifikation) beruhen auf fragwürdigen Annahmen (kann es wirklich sein, dass wir uns vergiften, wenn gleichzeitig unsere Lebenserwartung immer höher wird?) und können keine Evidenz für ihre Wirksamkeit vorweisen. Und Letzteres sollten wir immer verlangen, wenn es um die Therapie unserer Patientinnen und Patienten geht.

\section{Literatur}

1 Biedermann L, Rogler G, Zeitz J: «Mikrobiota-Tuning» en vogue: Von der Ernährung über Probiotika bis zur fäkalen Mikrobiota-Transplantation. Schweiz Z Ganzheitsmed 2016;29:DOI: 10.1159/000475586.

2 Frei P, Rogler G: Darm-Detoxifikation: Mythos oder sinnvolle Therapie? Schweiz Z Ganzheitsmed 2016;29:DOI: 10.1159/000475585.

3 Scharl M, Rogler G, Biedermann L: Anthocyane, Heidelbeeren und Curcuma: Wirksame Therapeutika bei Darmentzündungen? Schweiz Z Ganzheitsmed 2016;29:DOI: 10.1159/000475584. 